\title{
Controlling topology with strain
}

Large uniaxial strain drives a sequence of topological phase transitions in the quasi one-dimensional superconductor $\mathrm{TaSe}_{3}$, paving the way for the reversible control of surface spin currents.

\author{
Phil D. C. King
}

Concepts of topology have opened powerful routes to understand and classify the electronic band structures of materials. In a topological insulator, for example, an electronic band gap renders the material insulating in the bulk. A particular 'knotting' of the electronic wavefunctions, however, makes this distinct to an ordinary insulator, formally classified by different so-called topological invariants. ${ }^{1}$ While this is a bulk property, one of the most dramatic consequences is observed at the material's surfaces: metallic surface states are guaranteed to form, with a coupling between the electron spin and its momentum which is central to a number of proposed applications for spintronic devices. The topological origin of their surface states makes them largely resilient against the introduction of disorder and small changes in materials properties such as the charge carrier concentration. This is useful for fundamental study and for ensuring robust metallic surface conductivity, but makes it challenging to switch this on and off; something that would be required for a typical working device. Efforts to tune between topologically trivial and non-trivial phases tend to rely on changing material composition via chemical substitution. ${ }^{2}$ Now writing in Nature Materials, Chun Lin and colleagues ${ }^{3}$ have shown that it is possible to utilise uniaxial pressure to tune through a topological phase transition in the quasi one-dimensional superconductor TaSe ${ }_{3}$, in turn reversibly 'switching' its topological surface states on and off.

Pressure-tuning has long been established as an effective way to drive phase transitions. A challenge here, however, comes from the surface nature of the key experimental signature of the topologically non-trivial phase, which in general is extremely difficult to be isolated using bulk probes. Lin and colleagues therefore had to perform their pressure experiments in a way that would retain direct access to the surface electronic states of interest; they achieved this using uniaxial pressure. It was already known that uniaxial compression of single-crystal materials can lead to changes in lattice spacing on the percent scale, sufficient to drive dramatic modifications in their physical properties. ${ }^{4}$ Moreover, recent studies have successfully implemented capabilities for applying uniaxial pressure with simultaneous study of the materials' electronic structure using angle-resolved photoemission (ARPES), ${ }^{5-7}$ a surface-sensitive spectroscopic probe which has proved instrumental for studying topological materials. In fact, ARPES measurements had only just established the presence of surface electronic states consistent with a non-trivial band topology in $\mathrm{TaSe}_{3},{ }^{8}$ a compound comprised of stacks of onedimensional $\mathrm{TaSe}_{3}$ chains (Fig. 1). By probing the electronic structure along and across the chain direction using very highresolution spin-resolved ARPES experiments, Lin and colleagues were able to confirm the finding of a topological phase in unstrained $\mathrm{TaSe}_{3}$, directly visualising the spin-momentum locking which is a hallmark signature of its topological surface states.

More importantly, by applying tensile strains of up to ca. $2.5 \%$ along the chain direction, Lin and colleagues presented how the electronic structure evolves through a series of phase transitions. At a uniaxial strain approaching $1 \%$, the bulk bands which cross through each other (are 'inverted') to generate the topological phase in the unstrained compound become uncrossed (Fig. 1), and the spin-polarised topological surface states cease to exist. Additional bulk states still cross the Fermi level, however, making the material a topologically trivial semimetal. There is no longer a surface conduction channel derived from topological states, but there is still finite bulk conduction. This is why the phase transition observed here is difficult to be determined using transport measurements, underlining the importance of the surface-sensitive experimental probe used in this study. With further increase in strain, these bulk states too are pushed away from the Fermi level, mediating a transition to a (topologically trivial) insulating state. From electronic structure calculations, Lin and colleagues not only confirmed the experimentally-observed electronic structure evolution under tensile strain, but also predicted that by applying compressive strain, it should be possible to drive $\mathrm{TaSe}_{3}$ across another topological phase transition into a socalled weak topological phase, where the protected surface states exist only for certain surface orientations of the crystal. While it could not be realised experimentally, this prediction certainly motivates future experiments with strain cells optimised for applying compressive strains. ${ }^{7}$

While previous studies have shown that it is possible to use mechanical strain to modify the surface states of topological insulators, ${ }^{5}$ by a careful choice of a material sitting close to the borderline between several topologically distinct phases, Lin and colleagues have demonstrated that it is possible to deterministically, and reversibly, switch a material between topologically trivial and non-trivial phases. Exploiting this effect in technologies would still require developing scalable methods suited for device applications, but Lin and colleagues' findings represent an exciting proof of concept result, while already providing a platform for fundamental study. Indeed, many interesting questions remain. Is it possible to access the weak topological phase experimentally ${ }^{9}$ as mentioned above? Do the topological surface states of this bulk superconductor themselves support an elusive topological superconductivity, or do they simply coexist with a topologically-trivial superconductivity as in some other superconducting topological materials? ${ }^{10}$ Can strain tuning across the topological phase transitions shed light on this? Beyond this specific material, it is clear that uniaxial strain combined with advanced spectroscopic probes is rapidly becoming a promising tool for studying the electronic structure evolution of quantum materials, where many more discoveries can be expected. 
References:

1. Moore J. E. (2010). The birth of topological insulators. Nature 464, 194.

2. Xu S.-Y. et al. (2011) Topological Phase Transition and Texture Inversion in a Tunable Topological Insulator. Science 332, 560.

3. Lin et al. (2021) Nature Materials, in press (focus paper).

4. e.g. Steppke, A. et al. (2017) Strong peak in $\mathrm{T}_{c}$ of $\mathrm{Sr}_{2} \mathrm{RuO}_{4}$ under uniaxial pressure. Science 335 eaaf9398.

5. Flötotto D. et al. (2018) In Situ Strain Tuning of the Dirac Surface States in $\mathrm{Bi}_{2} \mathrm{Se}_{3}$ Films. Nano Lett. 18, 5628

6. Riccò S. et al. (2018) In situ strain tuning of the metal-insulator-transition of $\mathrm{Ca}_{2} \mathrm{RuO}_{4}$ in angle-resolved photoemission experiments. Nature Commun. 9, 4535.

7. Sunko et al. (2019) Direct observation of a uniaxial stress-driven Lifshitz transition in $\mathrm{Sr}_{2} \mathrm{RuO}_{4}$. npj Quantum Materials 4, 46.

8. Chen, C. et al. (2020) Observation of Topological Electronic Structure in Quasi-1D Superconductor TaSe 3 . Matter 3, 2055.

9. Hyun J. et al. (2020) A weak topological insulator state in quasi-one-dimensional superconductor TaSe 3 . arXiv:2009.05917.

10. Clark O. J. et al. (2018) Fermiology and superconductivity of topological surface states in PdTe 2. Phys. Rev. Lett. 120,156401

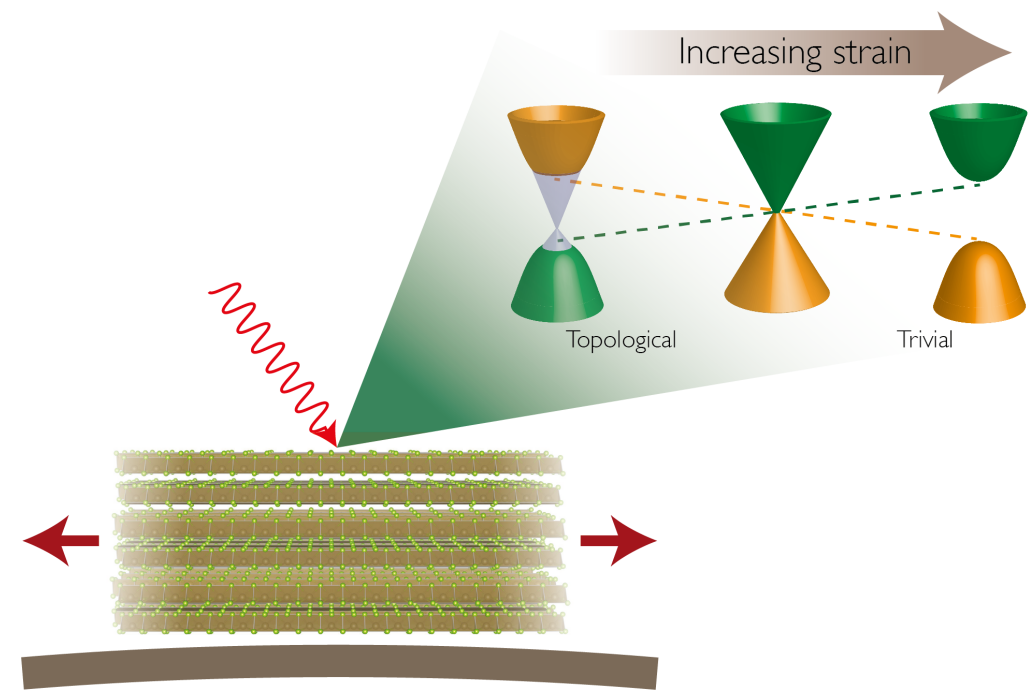

Figure 1 | Uniaxial strain tuning of a topological phase transition. Schematic of the bending platform device used to strain $\mathrm{TaSe}_{3}$ (bottom left). The quasi-1D material is oriented such that strain is applied along the chain direction, with the cleaved surface of the material accessible for ARPES measurements. This allows direct observation of the resulting topological phase transition which occurs when the large uniaxial tensile strain un-inverts the bulk bands, as shown in a simplified schematic (top right). 